\title{
TDAH y Dificultades de Aprendizaje en escritura: comorbilidad en base a la Atención y Memoria Operativa
}

\author{
Celestino Rodríguez ${ }^{1}$, David Álvarez ${ }^{1}$, Paloma González-Castro ${ }^{1}$, Jesús N. \\ García $^{2}$, Luis Álvarez ${ }^{1}$ José C. Núñez ${ }^{1}$, Julio A. González ${ }^{1}$ y Ana Bernardo ${ }^{1}$ \\ ${ }^{1}$ Universidad de Oviedo ${ }^{2}$ Universidad de León
}

\begin{abstract}
En esta investigación, se pretende conocer las diferencias entre alumnos con TDAH y dificultades de aprendizaje en escritura en una evaluación de atención, memoria operativa y estilos cognitivos. Se parte de una muestra de alumnos diagnosticados con TDAH, con edades relativas a prácticamente toda la etapa escolar obligatoria. Para, posteriormente, realizar una distinción entre alumnos TDAH con DA en escritura (DAE), TDAH sin DAE. Estas dos condiciones, se complementan con otro grupo de alumnos con DAE exclusivamente y un grupo control. Estos cuatro grupos, 59 alumnos con TDAH y DAE, 40 sólo con TDAH, 115 con DAE, y 124 en el grupo control. Forman una muestra de 339 sujetos, emparejados en cuanto a edad, género, CI y otras características educativas. Observando los resultados comparados por grupos de todas las medidas independientemente. Se concluye, que puede existir, un patrón de resultados más deficitario en pruebas de atención y memoria operativa en los niños con TDAH y DAE. Destacando una relación de comorbilidad entre ambos trastornos, descartando la hipótesis de fenocopia y corroborando otros estudios de DA en lectura y matemáticas. Se concluye con algunas consideraciones acerca del grado de dificultad de las tareas y su incidencia en el TDAH.
\end{abstract}

Palabras clave: TDAH, dificultades de aprendizaje, atención, memoria operativa.

ADHD and Writing learning disabilities: comorbidity in basis of attention and working memory. The aim of this study was to compare the differences between children with ADHD both with and without writing LD (WLD) and those with WLD, in the measurements of different constructs related to ADHD (selective and sustained attention, working memory and cognitive styles). Our sample comprised 339 students from $3^{\text {rd }}$ year of Primary school to $4^{\text {th }}$ year of Secondary School and consisted of four groups: 59 students diagnosed with ADHD and WLD, 40 with ADHD without WLD, 115 with WLD and/or low achievement (LA), and 124 normally achieving students formed the control group. All of the students ranged between eight and sixteen years old, matched in age and educational level. The results were observed independently between the groups in all the previously mentioned aspects. In conclusion, lower results were found in children suffering from ADHD and WLD as regards the attention and memory evaluation. Considering these results, we conclude by making a few remarks on the difficulty of the tasks and its effects on ADHD. Emphasizing comorbidity relation among both disorders, rejecting fenocopy hypothesis and corroborating other studies in reading and math.

Key words: ADHD, learning disabilities, attention, working memory.

Correspondencia: Celestino Rodríguez/Luis Álvarez Pérez. Área de Psicología Evolutiva y de la Educación. Departamento de Psicología. Universidad de Oviedo. Plaza Feijoo s/n. 33003 Oviedo. España. E-mail: rodriguezcelestino@uniovi.es; lalvarez@uniovi.es. 
Conocemos, en base a los estudios de revisión empírica y teórica aparecidos últimamente, el alto grado de solapamientos de dos de las dificultades más habituales en nuestras aulas y colegios, el Trastorno por Déficit de Atención e Hiperactividad (en adelante TDAH), y las Dificultades de Aprendizaje en escritura (en adelante DAE) (Miranda, Jarque y Tárraga, 2006; Reid, Trout y Schartz, 2005).

Por el contrario, resulta paradójico que a pesar de esa relación evidente, desconozcamos a nivel científico muchos elementos de esta conexión, así como una explicación empírica concreta (Barkley, 1996; Decker, McIntosh, Kelly, Nicholls y Dean, 2001; Kaplan, Dewey, Crawford y Wilson, 2001; Tabassam y Grainger, 2002). Además, se han encontrado pocos estudios que aborden el tema del TDAH desde la perspectiva de las DAE en escritura, o simplemente desde el enfoque de la escritura. Siendo estos escasos estudios, prácticamente inexistentes en español, lo que justifica el estudio que presentamos a continuación.

Para ejemplificar esta necesidad, sabemos por ejemplo, que un tercio de los niños con DA presentan a su vez algún subtipo del TDAH (Tabassam y Grainger, 2002). Además, se estima que entre el $19 \%$ y el $26 \%$ de personas con TDAH tienen algún tipo de DA. Todo ello evidencia el papel de los problemas atencionales en el ámbito escolar (Shalev y Tsal, 2003). En general, se considera que estos problemas, afectan a la función ejecutiva más que al factor de inteligencia, aspecto éste que no se ve influenciado por el TDAH. Por lo tanto, se estima que el TDAH mas que un problema de capacidad, es un problema de activación y de ejecución (Schuck y Crinella, 2005).

Por último, otros estudios consideran que la profunda influencia de los problemas de atención en las aulas, han hecho que el TDAH sea considerado un factor fundamental en las dificultades escolares (Decker et al., 2001). Además, se han diferenciado entre los síntomas de atención, que afectan preferentemente al trabajo en las clases y a la actuación académica (Chu, 2003). Y los síntomas de hiperactividad e impulsividad que afectan más directamente, aunque no exclusivamente, a la conducta de las personas con este trastorno (Tirado, Fernández y Hinojo, 2004).

Concretamente, en lo que hace referencia a las DAE, en una investigación con 119 niños entre 8 y 16 años, el $70 \%$ de los niños con TDAH presentaban una DA. De éstas, las DA escritura eran dos veces más frecuentes $(65 \%)$, que las DA en lectura, matemáticas u ortografía -deletreo- (Mayes, Calhoun y Crowell, 2000). En otro estudio realizado con 1004 personas, se concluye que el subtipo inatento dentro del TDAH, es el que presenta peores resultados en escritura comparado con los alumnos sin este trastorno (García et al., 2004, 2007; Presentación y Siegenthaler, 2005).

En general, la realidad escolar de los alumnos con TDAH y el estudio de las bases del problema, no han seguido la misma evolución que desde el plano médico. Incluso los recursos para abordarlo, desde el punto de vista psicopedagógico y de las DA, no están siendo del todo completos (Gregg, Coleman, Stennett y Davis, 2002). No 
obstante, con las base teóricas dominantes, se hacía necesario conocer la ejecución de estos alumnos en tareas de atención, memoria operativa, o el estilo reflexividad-impulsividad. Todas estas pruebas y estos constructos, guardan ciertas exigencias, en parte comparables, a las que requieren las tareas escolares. Y a su vez son interesantes, ya que están consiguiendo mayor protagonismo, y que están siendo reconceptualizadas en cuanto a su papel en este trastorno del desarrollo, el TDAH. Con estos datos y estos precedentes, se justifica el presente estudio. Sus conclusiones pueden aportar ideas importantes en todo este proceso, de reconceptualización y mejora de conexiones teóricas del TDAH con las DA en la escritura (Barkley, 2007).

\section{Objetivos}

Con todos los antecedentes comentados, se justifica el objetivo de este estudio. En concreto, lo enunciaríamos como el propósito de conocer el funcionamiento del ejecutivo mediante pruebas de atención, memoria operativa, y del estilo cognitivo reflexividad e impulsividad, en alumnos con TDAH y DAE. A su vez, se pretende conocer las diferencias en base a estos problemas. $\mathrm{Y}$ la hipótesis que se persigue contrastar, indica que estas medidas psicológicas serán más deficitarias en las personas con TDAH, que con DAE, y que, por supuesto, sus iguales sin dificultades. Es previsible, igualmente, que el grupo que presente TDAH y DAE será el que presente las mayores dificultades.

Asimismo, nos interesa conocer y poder descartar la hipótesis de fenocopia entre el TDAH y las DAE. Del mismo modo que ha sucedido en investigaciones precedentes con las DA en Lectura y Matemáticas (Fletcher, 2005). Aceptar la hipótesis de fenocopia, supone que los alumnos con TDAH y con DAE no presentan los déficit de ambos trastornos de forma más acusada. Por lo tanto, tienen los perfiles como suma de la combinación de los problemas de ambos trastornos.

Por otra parte, rechazarla supone que, este grupo con TDAH y DAE, tiene un patrón cognitivo único respondiendo a la idea de comorbilidad, interrelacionando todos esos problemas. Siendo mayor sus efectos negativos que la suma combinada de los mismos (Rucklidge \& Tannock, 2002).

\section{METODO}

\section{Participantes}

Los participantes en este estudio fueron un total de 339 alumnos y alumnas entre los 8 y los 16 años de edad. Cursaban estudios desde $3^{\circ}$ de Educación Primaria a $4^{\circ}$ de Educación Secundaria Obligatoria. Igualmente, la mayoría del grupo con TDAH procedía de la Asociación Leonesa de afectados por el Déficit de Atención e 
Hiperactividad (ALENHI). Todos los participantes estaban escolarizados en diversos centros educativos de toda la provincia de León.

Los participantes fueron distribuidos en 4 grupos atendiendo a su tipología; TDAH con DA en escritura (TDAHDA); TDAH sin DA en escritura (TDAHsDA); DA en Escritura (DAE), y un grupo control sin DA ni TDAH (SDA). En la tabla 1 se resume de forma esquemática la muestra, incluyendo las medias de edad y del Cociente Intelectual.

Tabla 1. Designación y características de la muestra

\begin{tabular}{lccccc}
\hline & \multicolumn{3}{c}{ Tipología } & \multirow{2}{*}{ Total género } \\
\cline { 2 - 5 } & DAE & SDA & TDAHDA & TDAHsDA & 216 \\
\hline Hombre & 63 & 73 & 50 & 30 & 123 \\
Mujer & 52 & 51 & 9 & 40 & 339 \\
Total tipología & 115 & 124 & 59 & 11.37 & \\
Media edad & 11.36 & 10.89 & 100.13 & 100.41 & \\
\hline \multirow{2}{*}{ Media CI (D.T.) } & 97.67 & 109.59 & $(13.11)$ & $(17.16)$ & \\
\hline
\end{tabular}

Todos los alumnos del grupo con TDAH fueron seleccionados atendiendo a su diagnóstico clínico profesional, ya fuera psiquiátrico o neuropsiquiátrico, en la mayoría de los casos, o psicológico. Asimismo, el 91.9\% de los participantes de este grupo, seguían un tratamiento de medicación que no fue interrumpida para realizar la evaluación, correspondiendo con las circunstancias habituales en que trabajan en el aula.

Finalmente, para corroborar el diagnóstico previo de este grupo, se pasó el cuestionario DSM-IV sobre TDAH a todos los participantes, tanto en el formato para padres como para profesores. Esta aplicación, nos sirvió para descartar casos de TDAH en los otros grupos de la muestra, como es el grupo control SDA y el grupo DA, así como para describir y definir de forma precisa la muestra. Y por último, se aplicó el cuestionario Five to Fifteen FTF (Kadesjö et al., 2004) para padres, a los alumnos del grupo TDAH, para conocer las peculiaridades de cada caso.

Con el fin de comprobar la homogeneidad de las muestras se realizó un análisis univariado de la varianza de los grupos en función de la edad; no apareciendo diferencias estadísticamente significativas, $\left[\mathrm{F}=1.277 ; \mathrm{p} \leq .279 ; \eta^{2}=.015\right]$. En cambio, al comparar los resultados del cuestionario DSM-IV para padres entre los cuatro grupos, resultó que existen diferencias estadísticamente significativas atendiendo a la tipología $\left[\lambda=.634 ; \mathrm{F}_{(3,16)}=6.113 ; \mathrm{p} \leq .000 ; \eta^{2}=.108\right]$. Las medias de cada uno de los grupos confirman los diagnósticos clínicos previos, puesto que los grupos con TDAH presentan medias superiores al resto de los grupos y al punto de corte.

En un paso posterior, se diferenció dentro del grupo con diagnóstico de TDAH a aquellos con DAE. Estos presentaban al menos dos años de retraso en la escritura (García et al., 2008), además de comprobar la presencia de un CI dentro de la normalidad (mayor de 85), y que no presentaran otras necesidades educativas especiales, 
así como una escolarización adecuada y normal. Con ello se identificaron dos subgrupos, uno de TDAH con DAE (TDAHDA), que cumplía los criterios de inclusión en los dos grupos y un grupo sin DAE (TDAHsDA).

Finalmente, tenemos otros dos grupos, uno con DAE exclusivamente, y otro de alumnos de rendimiento normal y sin TDAH. Primero, se obtuvo la opinión de profesores y psicopedagogos como un "screening” inicial. Después, se aplicó un baremo de escritura, para confirmar un retraso de al menos dos años en composición escrita y para la inclusión en el grupo DAE. El grupo control (SDA) se seleccionó teniendo en cuenta que no presentaban retraso en escritura, ni bajo rendimiento en escritura, ni DAE. Además, se tuvo en cuenta que no presentaran otras necesidades educativas especiales, además de exigirse un cociente intelectual dentro de la normalidad (mayor de 85).

\section{Instrumentos}

En lo que se refiere a las medidas relacionadas con el TDAH, se seleccionaron las pruebas a partir de la revisión bibliográfica, y teniendo en cuenta las diferentes líneas de investigación en torno al TDAH. Asimismo se tuvo en cuenta, la prueba piloto previa, así como las que mayor cantidad de información podrían aportar en función del objetivo del estudio. El resultado fue un abanico amplio de pruebas que detallamos a continuación, y que se pueden ver resumidas en la tabla 2.

\section{Evaluación de la atención}

En cuanto a la evaluación de la atención, se intentó usar una amplia batería de pruebas que se adaptaran al objetivo del estudio. Y que sirvieran para conocer también la relación entre diferentes pruebas diseñadas para medir el mismo tipo de atención, y contrastar resultados. Asimismo fueron todas ellas de aplicación colectiva.

En primer lugar, uno de los test de lápiz y papel sobre evaluación de la atención más usados en nuestro país actualmente, y que destaca por la cantidad de información que aporta, es el test d-2 de evaluación de la concentración y la atención selectiva (Brickenkamp, 2002).

Por otra parte, hemos considerado interesante, la utilización del test de las caras o test de percepción de diferencias de atención selectiva, debido a su tradición (Thurstone y Yela, 1979). Asimismo, otro test clásico y del mismo tipo, es el test de formas idénticas (Thurstone, 1986), pero aumentando el tiempo de 4 a 6 minutos. Esta variación, fue utilizada siguiendo la idea que se ha realizado en algún estudio con el test de las caras (Crespo-Eguílaz, Narbona, Peralta y Reparaz, 2006). Esta variación de la medida de atención selectiva permitiría, al aumentar su tiempo, una medida de la atención sostenida aproximada.

Por otra parte, hemos seguido la línea de investigación centrada en el factor de atención WISC-R (Wechler, 1994). Utilizando adaptaciones colectivas de diferentes 
sub-pruebas de esta batería, con el fin de evaluar el factor de atención-concentración del mismo (Miranda, Roselló y Soriano, 1998). Este factor, está compuesto por los subtest de Aritmética, Dígitos y Claves, aunque los resultados del primer estudio indicaban que la adaptación de la prueba de Aritmética no presentaba resultados de interés, por lo que fue eliminada. Además, hemos utilizado una adaptación colectiva de la sub-prueba de integración visual del ITPA (Kirk, McCarthy y Kirk, 1986).

Tabla 2. Aspectos evaluados e instrumentos utilizados en la evaluación

\begin{tabular}{|c|c|c|c|}
\hline Aspecto Evaluado & Instrumento & Tareas & Parámetros \\
\hline Factor de Atención & WISC-R (Wechler, 1994) & Dígitos y Claves & Puntuación directa \\
\hline Atención/Vigilancia & ITPA (Kirk, McCarthy \& Kirk, 1986) & Integración Visual & Puntuación directa \\
\hline Atención selectiva & Test de las Caras (Thurstone \& Yela, 1979) & Búsqueda visual & Puntuación directa \\
\hline Atención sostenida & Formas Idénticas (Thurstone, 1986) & Búsqueda visual & Puntuación directa \\
\hline $\begin{array}{l}\text { Atención selectiva y } \\
\text { concentración }\end{array}$ & d-2 (Brickenkamp, 2002). & Búsqueda visual & $\begin{array}{l}\text { Puntuación de atención sostenida. } \\
\text { Puntuación de atención selectiva. } \\
\text { Índice de concentración. } \\
\text { Puntuaciones parciales de aciertos, } \\
\text { omisiones y comisiones }\end{array}$ \\
\hline Estilos cognitivos I y II & EPPyFPE (García, Marbán \& de Caso, 2001) & $\begin{array}{l}\text { Reconocimiento y } \\
\text { rapidez cognitiva }\end{array}$ & $\begin{array}{l}\text { Puntuación de aciertos y errores, y } \\
\text { tiempo. Relacionados con un } \\
\text { índice de reflexividad- } \\
\text { impulsividad }\end{array}$ \\
\hline Memoria operativa & $\begin{array}{l}\text { Amplitud lectora (Elosúa, Gutiérrez, García } \\
\text { Madruga, Luque, \& Garate, 1996) }\end{array}$ & $\begin{array}{l}\text { Tarea adaptada a la } \\
\text { amplitud escritora. } \\
\text { Mantenimiento de } \\
\text { información }\end{array}$ & $\begin{array}{l}\text { Puntuación total conseguida y } \\
\text { nivel máximo alcanzado }\end{array}$ \\
\hline Funcionamiento ejecutivo & $\begin{array}{l}\text { Amplitud de escuchar (Pickering, Baqués, \& } \\
\text { Gathercole, 1999) }\end{array}$ & $\begin{array}{l}\text { Mantenimiento/ } \\
\text { procesamiento } \\
\text { simultaneo de } \\
\text { información }\end{array}$ & Puntuación \\
\hline $\begin{array}{l}\text { Trastorno por déficit de } \\
\text { atención con hiperactividad } \\
\text { (TDAH) }\end{array}$ & Cuestionario DSM-IV a padres (TDAH) & $\begin{array}{l}\text { Cuestionario } \\
\text { (18 ítems) }\end{array}$ & $\begin{array}{l}\text { Puntuación subtipo inatento, } \\
\text { hiperactivo-impulsivo y } \\
\text { combinado }\end{array}$ \\
\hline $\begin{array}{l}\text { Trastorno por déficit de } \\
\text { atención con hiperactividad } \\
\text { (TDAH) }\end{array}$ & Cuestionario DSM-IV a profesores (TDAH) & $\begin{array}{l}\text { Cuestionario } \\
\text { (18 ítems) }\end{array}$ & $\begin{array}{l}\text { Puntuación subtipo inatento, } \\
\text { hiperactivo-impulsivo y } \\
\text { combinado }\end{array}$ \\
\hline $\begin{array}{l}\text { Trastorno por déficit de } \\
\text { atención con hiperactividad } \\
\text { (TDAH) y problemas } \\
\text { relacionados }\end{array}$ & $\begin{array}{l}\text { Cuestionario Five to Fifteen para padres } \\
\text { (Kadesjo et al., 2004) }\end{array}$ & $\begin{array}{l}\text { Cuestionario } \\
\text { (179 ítems) }\end{array}$ & $\begin{array}{l}\text { Puntuación en } 9 \text { dominios o } \\
\text { problemas del TDAH }\end{array}$ \\
\hline \multicolumn{4}{|c|}{ Variables totales } \\
\hline \multirow{5}{*}{$\begin{array}{l}\text { Atención Sostenida } \\
\text { Atención Selectiva } \\
\text { Memoria Operativa } \\
\text { Ejecutivo -total de atención y memoria- } \\
\text { Factor de Atención }\end{array}$} & \multicolumn{3}{|c|}{ SUMA DE CONCENTRACIÓN DEL D2 y FORMAS IDÉNTICAS (6 min.) } \\
\hline & \multicolumn{3}{|c|}{ SUMA DE TOTAL DEL D2 y PERCEPCION DE DIFERENCIAS } \\
\hline & \multicolumn{3}{|c|}{ Suma ADAPTACIÓN DE Amplitud lectora, Amplitud de escuchar, $y$ Dígitos inverso } \\
\hline & \multicolumn{3}{|c|}{ Suma de atención sostenida, selectiva y memoria operativa } \\
\hline & \multicolumn{3}{|c|}{ Suma de Dígitos directo, total de claves e integración visual } \\
\hline
\end{tabular}

Con estos instrumentos, evaluamos un amplio campo de la atención como atención sostenida (con las limitaciones implícitas en el sistema de evaluación), y selectiva principalmente, así como, la capacidad de concentración.

Evaluación de la memoria operativa (MO) y estilos cognitivos

Sabemos que la relación entre la memoria operativa ó memoria de trabajo y la atención existe (Miranda y Meliá de Alba, 2005), y es indispensable realizar un 
evaluación de la misma en este estudio, en el que el TDAH es uno de los focos. También es cierto, que podríamos incluir en este apartado la sub-prueba de dígitos inversos del WISC-R, que hemos indicado anteriormente en la evaluación de la atención, aunque posteriormente veremos su relación dentro de lo que podemos denominar ejecutivo. Para este propósito de evaluación, utilizamos una adaptación colectiva de dos pruebas de evaluación del ejecutivo central, en concreto de memoria operativa o de trabajo, amplitud de escuchar (Pickering, Baqués y Gathercole, 1999) y amplitud lectora (Elosúa, Gutiérrez, García-Madruga, Luque y Garate, 1996). Consiguiendo así, ciertas medidas sobre mantenimiento y procesamiento simultáneo de información, y mantenimiento de la información, respectivamente.

Por otra parte, es indudable que interesaba conocer el estilo cognitivo reflexividad-impulsividad de los grupos y las tipologías que componen el presente estudio, para ello se utilizaron las pruebas de Estilos cognitivos I y II (EPPyFPE) (García, Marbán y de Caso, 2001). En general, los indicadores que han de considerarse para medir el grado de reflexividad-impulsividad (en adelante R-I) en Estilos cognitivos I son: el tiempo que se tarda en realizar la tarea; el número de aciertos; el número de errores cometidos. Si tomamos estas dos últimas variables en relación con la primera, vamos a obtener cuatro grupos de alumnos: los lentos y exactos (serían los reflexivos); los rápidos e inexactos (serían los impulsivos); los rápidos y exactos (tendrían un buen sistema de búsqueda), y los lentos e inexactos. Todo ello dependiendo de un índice de reflexividad-impulsividad (IRI).

$$
\mathrm{IRI}=\frac{\left(\mathrm{N}^{\mathrm{o}}\right. \text { Aciertos/30) }}{\text { Tiempo }}
$$

Consiste en un cociente considerando los aciertos y los errores sobre el total del tiempo. Para ello, en el numerador habrá que incluir el porcentaje de aciertos sobre el total de ítems ( $\mathrm{n}^{\circ}$ aciertos dividido entre 30 ítems). En el denominador figurará el tiempo que invirtió en la realización de la tarea.

En Estilos cognitivos II, las medidas tomadas son el número de aciertos; el número de errores y el sistema de búsqueda utilizado. Finalmente, se aplica una prueba de control de ortografía, para asegurarse de que lo que se obtiene tiene que ver con la reflexividad y no con el desconocimiento escrito de las palabras.

\section{Diseño}

Se utilizó un diseño experimental 4 X 1, con un grupo control. Uno de los factores era la presencia o no de TDAH y el segundo la presencia de DA en escritura, con lo que se formaban los cuatro grupos (TDAHsDA, TDAHDA y DA, así como el grupo control SDA). Se pretende, por tanto, ver las diferencias en las distintas variables 
dependientes (medidas de atención, memoria operativa, y estilos cognitivos) entre los grupos formados resultantes.

\section{Procedimiento}

Previamente al inicio del estudio, y una vez concluida la revisión bibliográfica sobre el tema y diseñado el estudio, se realizó un contacto previo con la Asociación Leonesa de Afectados por el Déficit de Atención e Hiperactividad (ALENHI). El fin de este contacto, era conseguir una muestra significativa de niños y niñas diagnosticados con TDAH, ya que sería muy difícil conseguirla en los colegios. Se envió una carta explicativa, a todas las familias de la asociación pidiendo su colaboración en el estudio. Respondiendo positivamente más de un centenar de familias, y firmándose con la asociación un convenio de investigación.

Seguidamente se iban haciendo grupos de 4 a 6 niños, a los cuales se les citaba en el local de dicha asociación, para realizar la evaluación. En la primera sesión se les entregaba a los padres los cuestionarios correspondientes, para que los devolvieran debidamente cumplimentados, y se tenía una breve entrevista con ellos. En esta primera sesión, de aproximadamente 50 minutos, se les aplicaban las pruebas de evaluación determinadas, de forma contrabalanceada, en ésta y en el resto de las sesiones hasta completar la evaluación.

Finalmente, se convenía con los padres el horario de las dos sesiones restantes, para concluir la evaluación del niño y recoger los cuestionarios. Se tuvo en cuenta que las sesiones no fueran en un horario posterior a las 7 de la tarde.

Una vez conocido el grupo de TDAH, interesaba conocer cuáles de estos alumnos presentaban DAE. Para ello, se aplicó un baremo basándose en una tarea de redacción de escritura, y buscando dos años de retraso con el mismo (productividad y/o coherencia). Teniendo en cuenta que, ese retraso, no es debido a un CI bajo, a una escolarización deficiente, ni a otra necesidad educativa especial.

Seguidamente, se procedió a la selección de los otros dos grupos, comparables en cuanto a curso y a edad al grupo con TDAH. Para ello, se contactó con varios centros educativos, de características similares, en cuanto a tipología y características demográficas, a los centros en los que estaban escolarizados los alumnos del grupo TDAH. De los centros que participaron, se solicitó de los maestros una primera apreciación sobre los alumnos con retraso en escritura. A estos alumnos se les estudió, con tareas de escritura, para determinar si se les ubicaban en el grupo de DAE o en el grupo control. Un vez seleccionados, se continuó con el mismo proceso de evaluación realizado en el anterior grupo, una evaluación a través de 3 sesiones y un contrabalanceo adecuado de las pruebas.

Por último, se procedió a la corrección de las pruebas y a la codificación de los datos; finalizando con la aplicación del baremo nuevamente para confirmar la 
composición correcta y definitiva de los grupos por tipología. A continuación se realizó el análisis de los datos y finalmente, se concluyó con la escritura del presente artículo.

\section{RESULTADOS}

Se utilizó el paquete estadístico SPSS 14.0 y 15.0, para la realización de los diferentes análisis, que se presentan seguidamente, atendiendo al constructo psicológico evaluado, es decir, atención, memoria operativa, estilos cognitivos y totales de atención.

En primer lugar, para asegurarse de que las variables son normales y poder realizar los análisis paramétricos, se comprobó la asimetría y la curtosis, indicando distribuciones normales de las variables. Con ello resuelto, se procedió a la realización de los análisis multivariados de la varianza. En general, se consideraron como factores inter-sujetos las cuatro tipologías (TDAHDA, TDAHsDA, DA y SDA), y como variables dependientes las medidas de los constructos en estudio (atención, memoria operativa, reflexividad-impulsividad).

\section{Resultados de atención, memoria operativa, y estilos cognitivos en función de la tipología}

Cuando se realizan los contrastes multivariados de la varianza en función de la tipología, se obtienen datos estadísticamente significativos, siendo el tamaño del efecto grande $\left[\lambda=.503 ; \mathrm{F}_{(3,102)}=1.931 ; \mathrm{p} \leq .000 ; \eta^{2}=.205\right]$. Las pruebas de los efectos inter sujetos resultan estadísticamente significativas en un gran número de variables relacionadas con atención, memoria operativa, y estilos cognitivos, tal y como se muestra en la tabla 3 .

De todas estas medidas, destaca sobre todo la medida de concentración del d-2, y la prueba de dígitos. Así como era esperable, las cinco medidas totales de atención sostenida, atención selectiva, memoria operativa, ejecutivo -total de atención y memoriay factor de atención. Todo ello parece interesante, por la importancia que tendrán posteriormente en las conclusiones relativas al diagnóstico. También las diferencias estadísticamente significativas son altas en estilos cognitivos. Analizaremos, por separado y en función de los contrastes post hoc, los resultados parciales de atención y memoria en las diferentes pruebas, por un lado, y los totales de atención y memoria operativa y estilos cognitivos por otro. Se puede ver un resumen de estos contrastes en la tabla 4.

Resultados parciales en las diferentes pruebas de atención y memoria operativa

En cuanto a las medidas parciales de las diferentes pruebas de atención, los contrastes post hoc, muestran diferencias estadísticamente significativas en un gran 
número de variables. Destacando por encima del resto, las diferencias entre el grupo SDA vs., TDAHDA, y las diferencias entre DA vs., SDA. La tendencia observada muestra que las medidas parciales no aportan información relevante, y debemos fijarnos en los totales de las medidas en el D2. Por norma general, vemos cómo el grupo con DA alcanza valores bajos en estas medidas, por debajo incluso del grupo TDAHDA.

Tabla 3. Modelo lineal general considerando como factor la tipología y como variables dependientes las medidas de atención, memoria operativa MO y estilos cognitivos

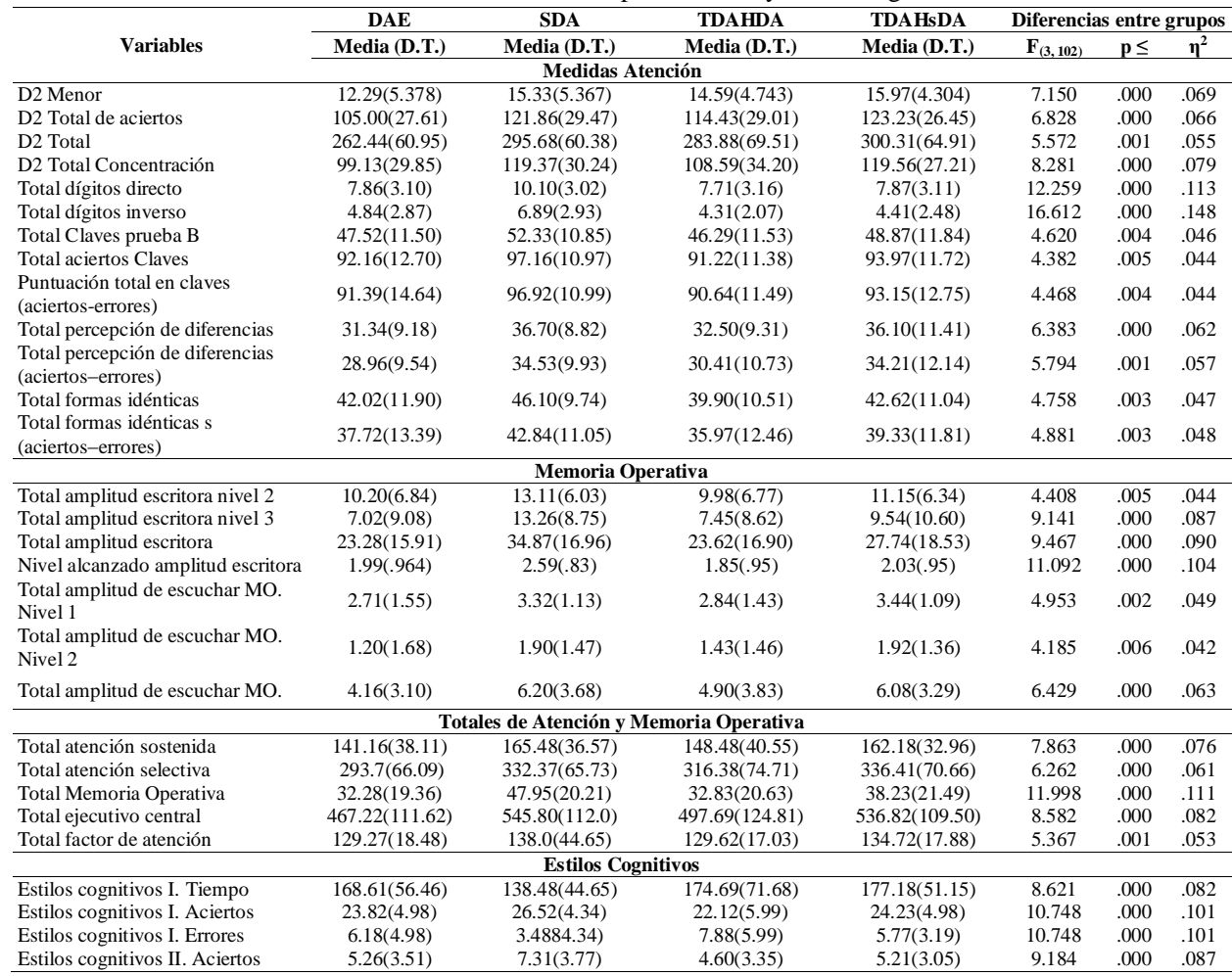

*Sólo se presentan datos estadísticamente significativos ( $\mathrm{p}<.05)$ o próximos a la significatividad estadística.

$* * \eta^{2}$ (estadístico eta-cuadrado)=estima el tamaño del efecto. La regla de Cohen (1988) asigna=.01-.06 (efecto pequeño); >.06-.14 (efecto medio);>.14 (gran efecto)

Igualmente, en los contrastes post hoc, aparecen diferencias estadísticamente significativas en dígitos del WISC. En concreto, se puede observar cómo las diferencias entre los dos grupos con TDAH en Dígitos inverso prácticamente son inapreciables, dando muestras de deficiencias en la memoria operativa en los alumnos con este trastorno del desarrollo. 
Tabla 4. Contrastes post hoc que son significativos, en los análisis multivariados, de las medidas de atención, memoria operativa y estilos cognitivos (por tipología)

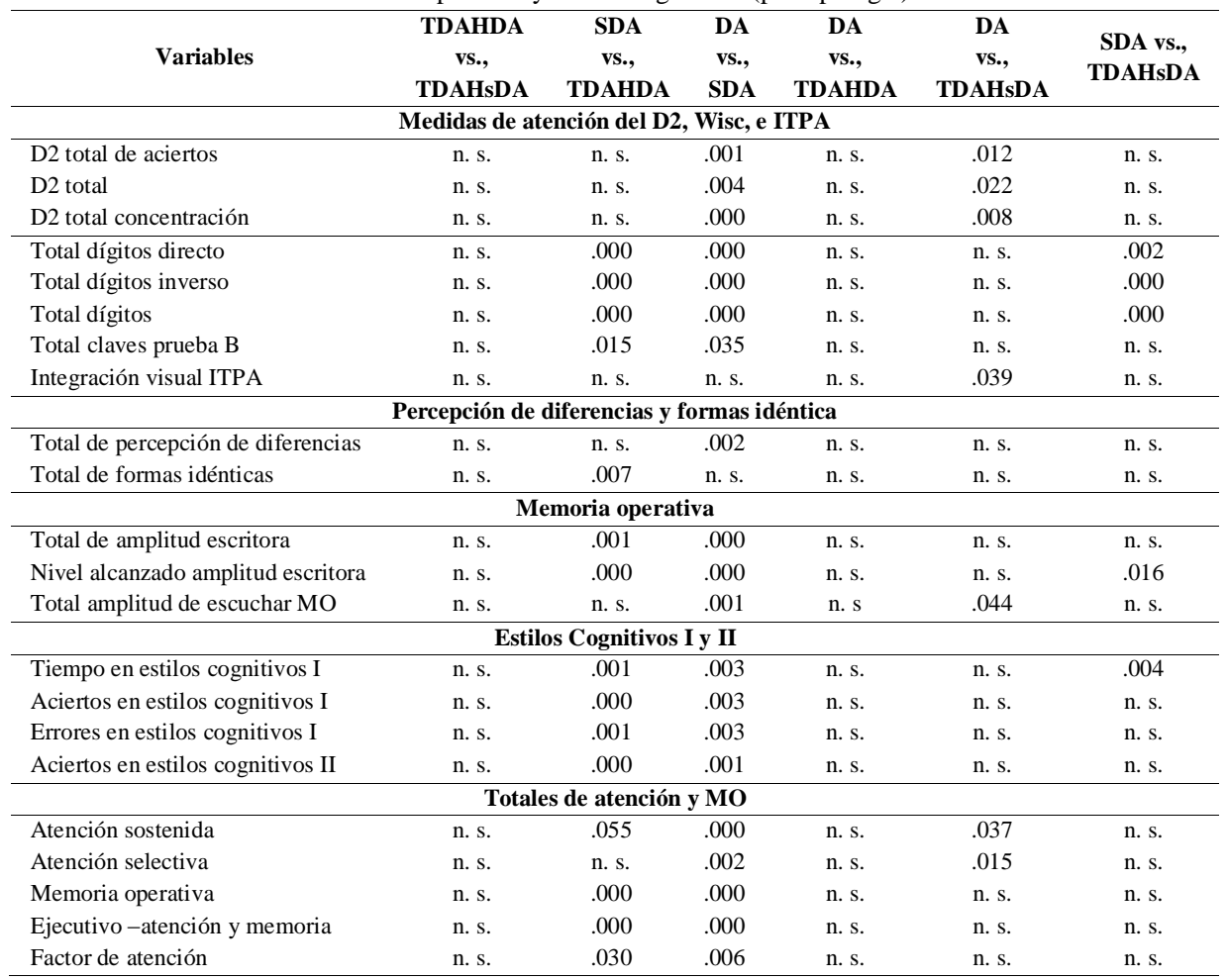

Figura 1. Medidas de Percepción de Diferencias y Formas Idénticas

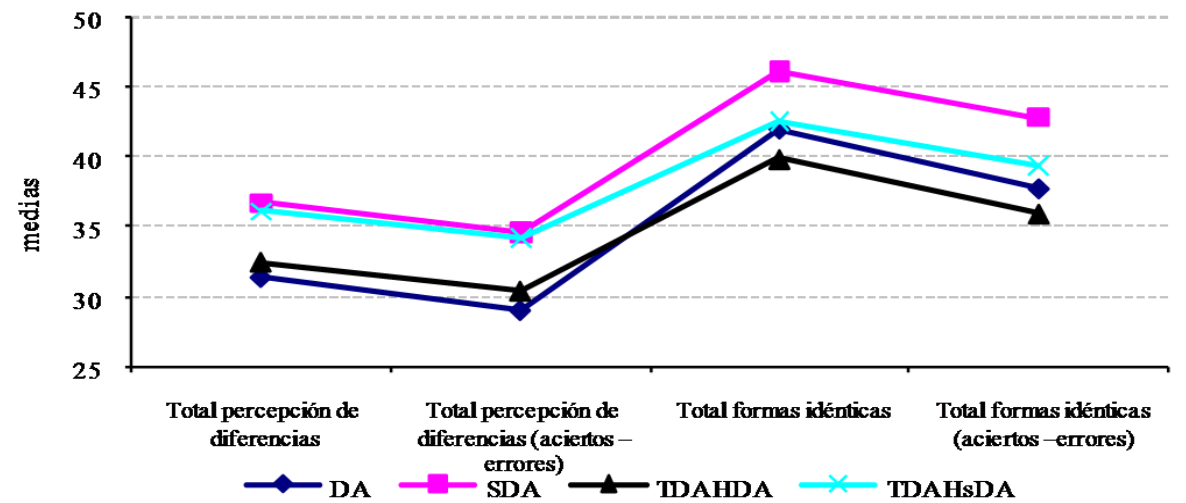

Dos pruebas de gran tradición en cuanto a evaluación de atención selectiva son Percepción de Diferencias y Formas Idénticas que aportan información concluyente. 
La atención durante un período prolongado como en Formas Idénticas destaca claramente las diferencias entre tipologías como podemos ver en la figura 1. Así como, también, el tener en cuenta los errores en la tarea, hace que las diferencias entre los grupos con TDAH sean más nítidas.

Figura 2. Resultados de las diferentes tipologías en las pruebas de Memoria Operativa (MO)

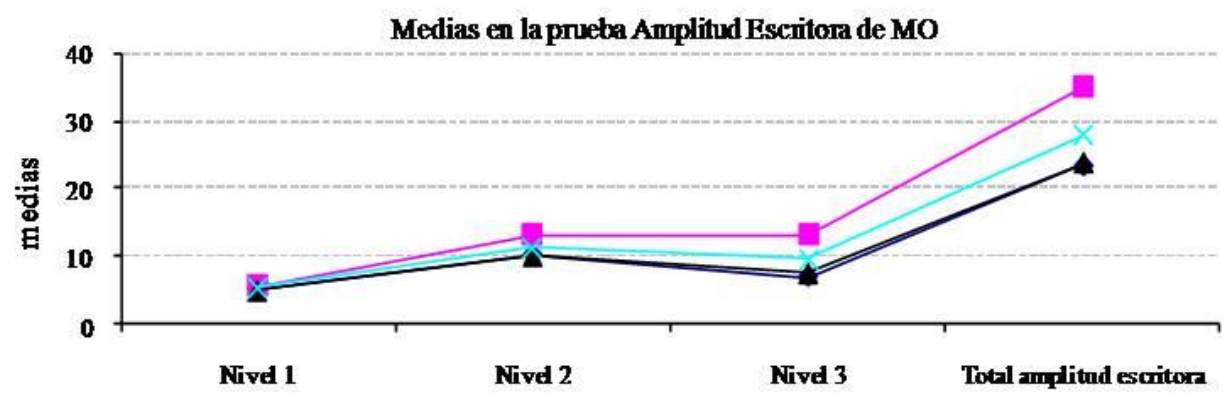

Totales de nivel en amplitud de escucharMO.

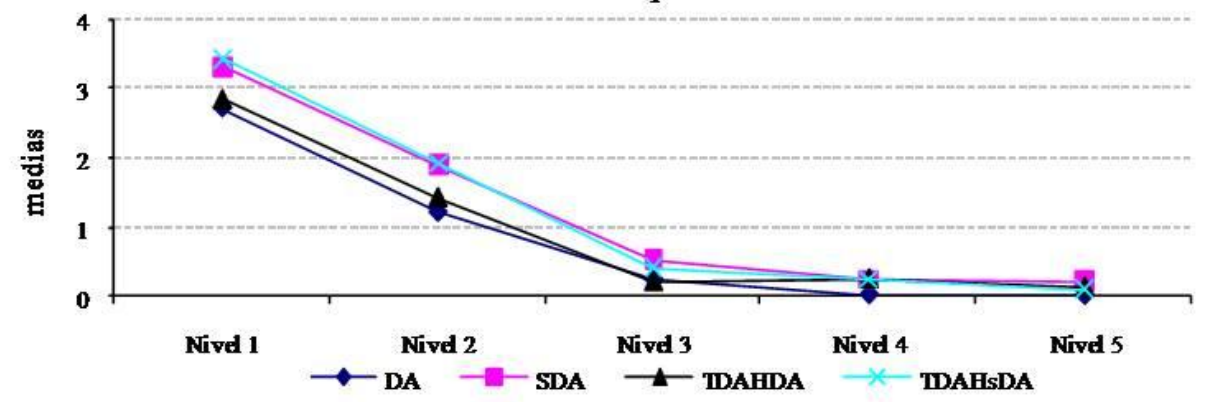

En cuanto a las variables de MO, es interesante ver los resultados obtenidos con respecto a las dos pruebas utilizadas. Cuando la tarea va necesitando mayor esfuerzo de memoria de trabajo, es decir, se va incrementando en dificultad, en los contrastes post hoc las diferencias entre grupos se hacen estadísticamente más significativas (Figura 2). Del mismo modo, cuando la tarea es demasiado compleja, esas diferencias no son significativas. Lo vemos en total de amplitud escritora; nivel alcanzado amplitud escritora; total amplitud de escuchar MO.

Finalmente, en los totales de las diferentes medidas globales de atención y memoria operativa los contrastes post hoc muestran diferencias estadísticamente significativas sobre todo cuando se tienen en cuenta la suma de MO y atenciónejecutivo- (Figura 4). En síntesis, se dan diferencias estadísticamente significativas por tipología y son de las más concluyentes por el número de puntuaciones que engloban, y 
el peso estadístico que tienen, estas son la variable atención sostenida; atención selectiva; memoria operativa; ejecutivo y factor de atención.

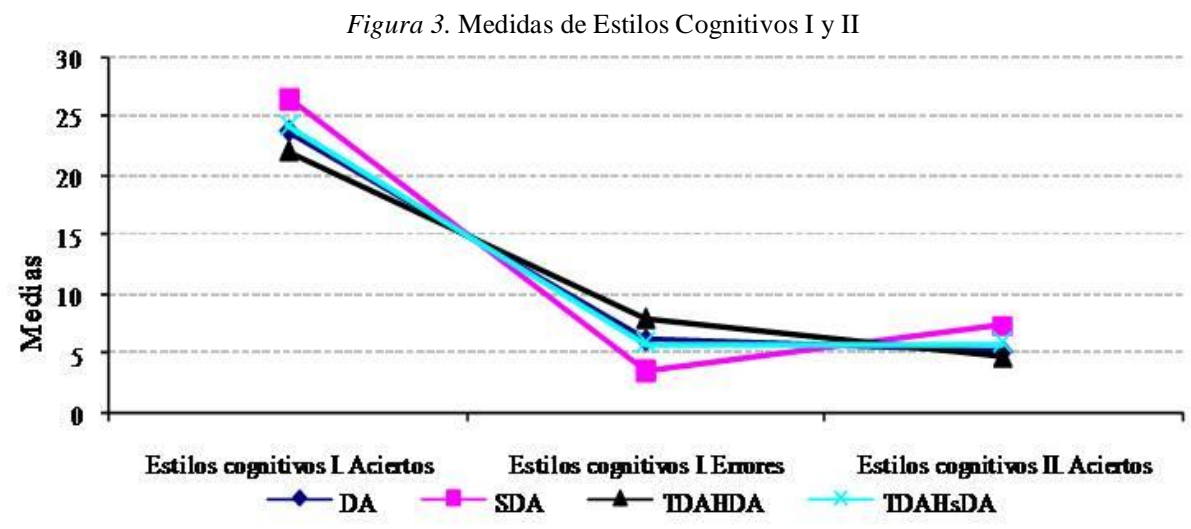

Figura 4. Medidas totales de Atención y Memoria Operativa dependiendo de la tipología

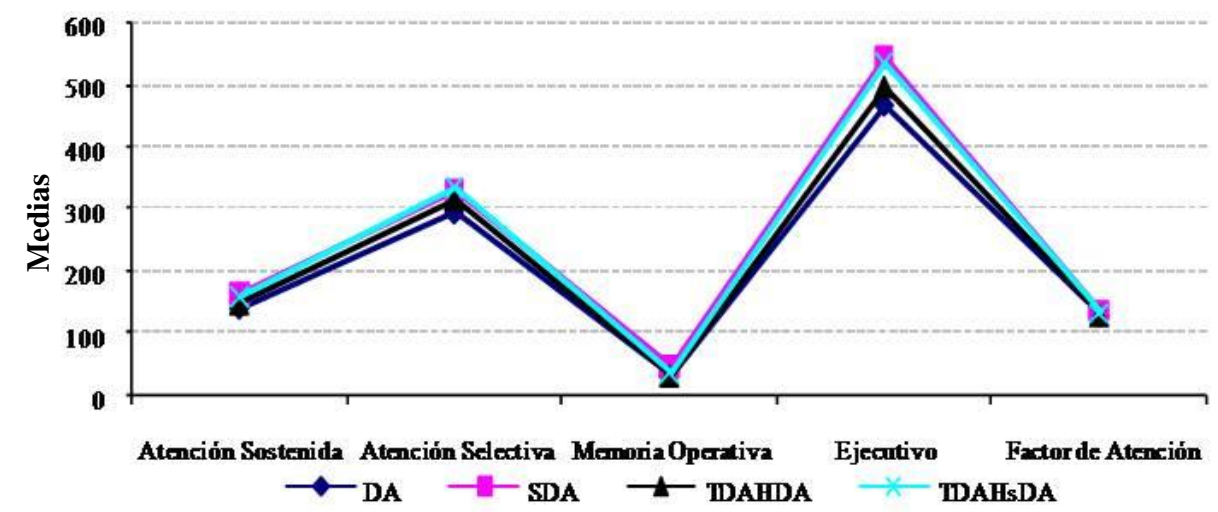

DISCUSION

Podemos concluir el estudio, indicando que el objetivo propuesto se ha conseguido de forma razonable. Puesto que hemos podido analizar y contrastar diferentes medidas de atención, MO y estilos cognitivos, en alumnos bajo las condiciones de TDAH y DAE, y todos ellos comparados con un grupo control. Las hipótesis se confirman con los totales, pero no con las medidas aisladas, y desde luego no con la claridad esperada. En general, la variedad de pruebas utilizadas, y los 
resultados obtenidos con los análisis, proporcionan elementos de interés en la relación del TDAH y las DAE.

Concretamente, merece la pena resaltar que, las hipótesis se cumplen cuando nos fijamos en los totales hallados como compendio de pruebas, y no cuando analizamos esas pruebas por separado. Y lo cierto es que se intuye una progresión clara, con un mayor poder discriminativo, cuanto más medidas aunamos y tengamos en cuenta. Es por esto, que cuando a las medidas de atención sostenida, selectiva y MO, las conjuntamos en una medida llamada ejecutivo. Ésta última, discrimina más eficazmente que cuando utilizamos cada una por separado. Anticipando y ejemplificando una conclusión clara, en torno a la necesidad de un diagnóstico comprehensivo y completo en el continuo del TDAH (Gillbert et al., 2004).

Por una parte, se hipotetizaba que la atención -concentración- fuera menor en los grupos con TDAH. Pues bien, esto es cierto para las medidas globales del D-2 (Brickenkamp, 2002). En ellas, se observan menores niveles de atención en los dos grupos con TDAH - con y sin DAE-, pero también se observa en el grupo con DAE exclusivamente. En cambio, no hay diferencias en las medidas parciales de atención, lo que hace que el grupo sin DAE y sin TDAH se comporte de forma similar al grupo con TDAH sin DAE. Esto es muy interesante, puesto que indica la necesidad de utilizar baterías de pruebas, que sí discriminan, más que pruebas aisladas. Además nos propone una tipología específica de pruebas para medir la atención. En las que existan una serie de parámetros a tener en cuenta, como por ejemplo omisiones y comisiones.

Las medidas de atención selectiva, apenas han aportado información relevante. Excepto en lo que hace a la modificación introducida -prolongación del tiempo para medir concentración, y medida de la inhibición o impulsividad de errores (Barkley, 2006)-; que entonces las convierte en muy discriminativas. Pasando a atención sostenida, esta parece que sí que es relevante. Lo que encaja con estudios previos, sobre la importancia de tareas prolongadas para medir el déficit de atención y el TDAH (Álvarez, González-Castro, Núñez, González-Pienda y Bernardo, 2008; Crespo-Eguílaz, Narbona, Peralta y Reparaz, 2006).

En cuanto a las medidas de estilos cognitivos, parece ser que no se confirmaron las hipótesis claramente, de peor ejecución en los grupos con TDAH. Sin embargo, han proporcionado información sobre la lentitud del procesamiento $-\mathrm{y}$ no el dato de rapidez reflejado por la impulsividad-, tanto en el grupo con el TDAH, como del grupo con DAE, que le sigue en cuanto a lentitud. La cuestión es que este tiempo mayor, dedicado por estos dos grupos, no ha sido productivo. Es decir, presentan muchos errores y pocos aciertos, participando ambos del grupo lentos-inexactos (Frazier, Youngstrom, Glutting y Watkins, 2007).

Por el contrario, se confirman los déficits en $\mathrm{MO}$, relacionados con el ejecutivo central en el grupo con TDAH. Coincidente con los déficits ejecutivos que 
refieren los estudios actuales. Cabe destacar los problemas de este grupo, sobre todo en dígitos inversos y considerando el total- p. ej., en dígitos del WISC- (Miranda et al., 1998). Este patrón se repite en el resto de medidas de MO, influidas negativamente a su vez por las DAE. Quizás perjudicada por el tipo de tarea relacionada con la escritura (Vandemberg y Swanson, 2007). Por último, y en relación a esto, parece importante el grado de dificultad de la tarea a la hora de discriminar por grupos y ver sus diferencias (Nigg, 2005). Cuando una tarea es demasiado sencilla o demasiado complicada, ésta no discrimina entre grupos con TDAH. Pero cuando esta complejidad, es adecuada, se puede ver la deficiencia en MO perfectamente (Jacobson y Kikas, 2007).

Por último, en lo que se refiere a las medidas totales relacionadas con el TDAH. La influencia de los totales de atención y MO, indican que el mayor efecto fue el funcionamiento del ejecutivo. Quizás sea este compendio de atención y MO, el que haga que se hagan evidentes tanto los problemas relacionados con las DAE como con TDAH, en comparación con los alumnos sin TDAH ni DA. Estos resultados totales sobre atención y MO, parecen indicar el sentido de todas estas dificultades en un continuo, y dando pistas sobre la comorbilidad de las condiciones de este estudio, DAE y TDAH (Fletcher, 2005).

En conclusión, podemos decir que los alumnos con TDAH se diferencian de los de DAE, sobre todo en atención sostenida. Y que los de SDA, se diferencian del resto en casi todas las medidas totales, especialmente en estilos cognitivos y atención sostenida. Además, los alumnos con TDAH y DAE a la vez, se diferencian de los alumnos con sólo DAE en el funcionamiento del ejecutivo, cuando sumamos los totales de atención y memoria. Por otra parte, los alumnos con TDAH sin DA se diferencian de los alumnos con DAE en atención sostenida y selectiva, principalmente.

En segundo lugar, en referencia a alumnos con TDAH, es interesante contrastar la importancia creciente del déficit en la atención sostenida y el mantenimiento de la concentración, en comparación a la atención selectiva, por ejemplo. Asimismo, los resultados provenientes de la conjunción de MO y atención, muestra un vínculo entre estos dos constructos, a estudiar en el futuro del TDAH (Harder, 2007).

En tercer lugar, podemos decir que las DAE y el TDAH comparten algunos elementos deficitarios en su funcionamiento cognitivo, sobre todo las referidas a la atención y MO. Si bien, las explicaciones de ambas limitaciones puede que procedan de campos diferentes. Para el caso de las DAE, el uso ineficiente de la recursividad de los procesos puede ser la clave; en cambio, en el caso del TDAH, puede ser el ejecutivo (Álvarez et al., 2008; García et al., 2007).

Asimismo, se anticipa que las dificultades de las dos condiciones, TDAH y DAE interaccionan. Ello se traduce en un perfil único de sus tareas de atención, estilos cognitivos y MO, diferente al grupo control, y al grupo con TDAH o DAE exclusivamente. Esta idea sugiere que descartaría la hipótesis de fenocopia (Fletcher, 
2005), y lo que es más importante, aporta razones para pensar que ambos problemas tienen una relación de comorbilidad.

Por último, se ha de indicar que el presente estudio presenta las limitaciones propias de una evaluación de atención y MO de forma colectiva, o con medidas poco contundentes de atención sostenida. Por lo tanto, no es lo mismo que una evaluación individualizada y clínica. Por el contrario, tiene la ventaja de la relevancia educativa y de su aplicabilidad en el contexto natural del aula. Siendo este tipo de estudios necesarios aunque no suficientes.

Asimismo, se sugiere la necesidad de estudios adicionales considerando grupos de edad en específico, o el género, controlando los subtipos del TDAH, o controlando los niveles de atención o de MO. Además, son necesarios estudios de intervención que permitan comprobar, en qué medida, al mejorar una variable podemos incidir en aspectos esenciales deficitarios del TDAH o de las DAE (González-Castro, Álvarez, Núñez, González-Pienda y Álvarez, 2008). Las futuras investigaciones tienen aquí una primera piedra sobre las relaciones entre el TDAH y las DAE. De todas formas, la aplicabilidad y las conclusiones pueden aportar algunas ideas clave de cara a ello. Algunas de estas ideas están siendo contrastadas actualmente, si bien es ingente el programa de investigación que se abre y queda por cubrir.

\section{REFERENCIAS}

Álvarez, L., González-Castro, P., Núñez, J.C., González-Pienda, J.A. y Bernardo, A. (2008). Evaluación y control de la activación cortical en los déficit de atención sostenida. International Journal of Clinical and Health Psychology, 8(2), 509-524.

Barkley, R.A. (1996). Attention-deficit hyperactivity disorder. In: Mash, E.J. and Barkley, R.A. (Eds.), Child psychopatology (pp. 63-112). New York: Guilford Press.

Barkley, R.A. (2006). Attention-deficit hyperactiviy disorder: A handbook for diagnosis and treatment $\left(3^{\text {rd }}\right.$ ed.). New York: Guilford.

Barkley, R.A. (2007). School Intervention for Attention Deficit Hyperactivity Disorder: Where to From Here? School Psychology Review, 36(2), 279-286.

Brickenkamp, R. (2002). D-2. Test de atención. Madrid: TEA Ediciones.

Chu, S. (2003). ADHD part two: evaluation and intervention. International Journal of Therapy and Rehabilitation, 10(6), 254-263.

Crespo-Eguilaz, R., Narbona, J., Peralta, F. y Reparaz, R. (2006). Medida de atención sostenida y del control de la impulsividad en niños: nueva modalidad de aplicación del Test de Percepción de Diferencias "Caras". Infancia y Aprendizaje, 29(2), 219-232.

Decker S.L., McIntosh D.E., Kelly A.M., Nicholls S.K. y Dean R.S. (2001). Comorbidity among individuals classified with attention disorders. International Journal of Neuroscience, $110,43-54$.

Elosúa, M.R., Gutierrez, F, García-Madruga, J.A., Luque, J.L. y Gárate, M. (1996). Adaptación española del "Reading Span Test" de Daneman y Carpenter. Psicothema, 8(2), 383-395.

Fletcher, J.M. (2005). Predicting Math Outcomes: Reading predictors and Comorbidity. Journal of Learning Disabilities, 38(4), 308-312. 
Frazier, T.W., Youngstrom, E.A., Glutting, J.J. y Watkins, M.W. (2007). ADHD and Achievement: Meta-Analysis of the Child, Adolescent, and Adult Literatures and a Concomitant Study With College Students. Journal of Learning Disabilities, 40(1), 4965.

García, J.N., Marbán, J.M. y de Caso, A. (2001). Evaluación colectiva de los procesos de planificación y factores psicológicos de la escritura (EPP y FPE). En J.N. García: Dificultades de aprendizaje e intervención psicopedagógica (pp. 151-155). Barcelona: Ariel.

García, J.N., Marbán, J.M., Rodríguez, C., de Caso, A.M., Fidalgo, R., Arias-Gundín, O., González, L., Martínez-Cocó, B., Pacheco, D.I., Robledo, P., Díez, C. y Álvarez, M. L. (2008). Datos descriptivos sobre solapamiento de problemas atencionales y de hiperactividad e impulsividad en base a la escritura en alumnos con dificultades de aprendizaje. Boletín de Psicología, 91, 7-26.

García, J.N., Rodríguez, C., de Caso, A. M., Fidalgo, R., Arias-Gundín, O., González, L., y Martínez-Cocó, B., (2007). El Trastorno por Déficit de Atención e Hiperactividad (TDAH), diferencias entre los diferentes subtipos en la composición escrita. Análisis y Modificación de conducta, 33(149), 369-384.

García, J.N., Rodriguez-Pérez, C., de Caso, A., Fidalgo, R., Arias-Gundín, O., Gonzalez L., Martinez-Cocó, B., Pina, M.J., y López-Campelo, B. (2004). ¿Es posible diferenciar los problemas atencionales y de hiperactividad e impulsividad en base a los niveles de composición escrita?. IV Congreso Internacional de Psicología y Educación: Calidad Educativa. Universidad de Almería.

González-Castro, P., Álvarez, L., Núñez, J.C., González-Pienda, J.A., y Álvarez, D. (2008). The application of a computerised strategy to teach and learn mathematics in primary education. European Journal of Education and Psychology, 1(1), 21-38.

Gregg, N., Coleman, C., Stennett, R.B. y Davis, M. (2002). Discourse Complexity of College Writers with and Without Disabilities: A Multidimensional Analysis. Journal of Learning Disabilities, 35(1), 23-38.

Harder, L.L. (2007). The relation between executive functions and written expression in college students with attention deficit hyperactivity disorder. Dissertation Abstract International Section A: Humanities and Social Sciences, 68(1), 82-92.

Jakobson, A. y Kikas, E. (2007). Attention-Deficit/Hyperactivity Disorder With and Without Comorbid Learning Disabilities. Journal of Learning Disabilities, 40(3), 194-202.

Kadesjö, B., Janols, L.O., Korkman, M., Mickelsson, K., Strand, G., Trillingsgaard, A. y Gillbert, C. (2004). The FTF (Five to Fifteen): the development of a parent questionnaire for the assessment of ADHD and comorbid conditions. European Child \& Adolescent Psychiatry, 13(3), 3-13.

Kaplan, B.J., Dewey, D.M., Crawford, S.G. y Wilson, B.N. (2001). The Term Comorbidity Is Of Questionable Value in Reference to Developmental Disorders: Data and Theory. Journal of Learning Disabilities, 34(6), 555-565.

Kirk, S., McCarthy, J. J. y Kirk, W.D. (1986). Test Illinois de Aptitudes Psicolingüisticas. Madrid: TEA Ediciones.

Mayes, S.D., Calhoun, S.L. y Crowell, E.W. (2000). Learning Disabilities and ADHD: Overlapping Spectrum Disorders. Journal of Learning Disabilities, 33(5), 417-424.

Miranda, A. y Meliá de Alba, A. (2005). Psicología de la Instrucción: Mejora del proceso de E/A de los niños con TDAH. ler Congreso Nacional de TDAH. Valencia; Marzo de 2005.

Miranda, A., Jarque, S. y Tárraga, R. (2006). Interventions in School settings for Students with ADHD. Exceptionality, 14(1), 35-52. 
Miranda, A., Roselló, B. y Soriano, M. (1998). Estudiantes con Deficiencias Atencionales. Valencia: Promolibro.

Nigg, J.T. (2005). Attention, task difficulty, and ADHD. British Journal of Developmental Psychology, 23, 513-516.

Pickering, S.J., Baqués, J. y Gathercole, S.E. (1999). Batería de Test de Memoria de Treball. Barcelona: Laboratori de Memoria de la Universitat Autónoma de Barcelona (versión catalana de S. Pickering y S. Gathercole, Working memory Battery (1997). No comercializada .

Presentación, M.J. y Siegenthaler, R. (2005). Problemática asociada al TDAH subtipo combinado en una muestra escolar. Infancia y Aprendizaje, 28(3), 261-275.

Reid, R., Trout, A.L. y Schartz, M. (2005). Self-Regulation Interventions for Children With Attention Deficit/Hiperactivity Disorder. Exceptional Children, 71(4), 361-377.

Rucklidge, J.J. y Tannock, R. (2002). Neurospychological Profiles of Adolescents with ADHD: Effects os Reading Difficulties and Gender. Journal of Child Psychology \& Psychiatry \& Allied Disciplines, 43(8), 988-1003.

Schuck, E.B. y Crinella, F.M. (2005). Why children with ADHD Do Not Have Low Iqs. Journal of Learning Disabilities, 38(3), 262-280.

Shalev, L. y Tsal, Y. (2003). The Wide Attentional Window: A Major Deficit of Children with Attention Difficulties. Journal of Learning Disabilities, 36(6), 517-527.

Tabassam W. y Grainger J. (2002). Self-concept, attributional style and self-efficacy beliefs of students with learning disabilities with and without attention deficit hyperactivity disorder. Learning Disability Quarterly, 25, 141-151.

Thurstone, L.L. (1986). Formas Idénticas. Madrid: TEA.

Thurstone, L.L. y Yela, M. (1979). Percepción de diferencias (caras). Madrid: TEA.

Tirado, J.L., Fernández F.D., y Hinojo, F.J. (2004). Trastorno por déficit de atención con hiperactividad: intervención psicopedagógica. Psicothema, 16(3), 408-414.

Vanderberg, R. y Swanson, H.L. (2007). Which componentes of working memory are important in the writing process? Reading and Writing: An Interdisciplinary Journal, 20, 721-752.

Wechsler, D. (1994). Manual for the WISCR-R. Nueva York: Psychological Corporation.

Recibido: 5 de enero de 2009

Recepción Modificaciones: 6 de mayo de 2009

Aceptado: 10 de junio de 2009 